\title{
Comparative Analysis of Postoperative Complications after Cytoreductive Surgery and HIPEC in Gastric Cancer
}

\author{
Felix Merboth Sebastian Garcia Janusz v. Renesse Marius Distler \\ Thilo Welsch Jürgen Weitz Daniel E. Stange \\ Department of Visceral, Thoracic and Vascular Surgery, University Hospital Carl Gustav Carus, Medical Faculty, \\ Technische Universität Dresden, Dresden, Germany
}

\section{Keywords}

Gastric cancer - Peritoneal carcinomatosis · Hyperthermic intraperitoneal chemotherapy · Cytoreductive surgery · Complications

\begin{abstract}
Introduction: Patients with advanced gastric cancer (AGC) frequently show peritoneal carcinomatosis $(P C)$. $P C$ reduces life expectancy and quality of life. Cytoreductive surgery (CRS) with hyperthermic intraperitoneal chemotherapy (HIPEC) has been shown to improve overall survival. Nevertheless, it has been reported that CRS and HIPEC are accompanied by an increase in postoperative complications. The purpose of this study was to investigate the complications associated with CRS and HIPEC and overall and disease-free survival. Methods: Patients with AGC and PC, who received complete CRS and HIPEC, were included in the HIPEC group $(n=15)$. Patients with AGC but without PC, who received resection of the primary tumor alone, constituted the control group $(n=43)$. Results: Patients enrolled in the HIPEC group presented with a median $\mathrm{PCl}$ of 7 . In comparison with the control group, no differences were found in patient characteristics, risk factors, pathological findings, and operative procedures. Twenty-five percentage of the patients in both groups suffered from serious postoperative complications $(C D C \geq 3 a)$. Surgical and medical complications, rate of reoperation, and mortality did not differ. Also, the recurrence pat-
\end{abstract}

karger@karger.com www.karger.com/ort

Karger GOPEN ACCESS
(C) 2021 The Author(s)

Published by S. Karger AG, Basel

This article is licensed under the Creative Commons Attribution 4.0 International License (CC BY) (http://www.karger.com/Services/ OpenAccessLicense). Usage, derivative works and distribution are permitted provided that proper credit is given to the author and the original publisher. tern, median survival, and 1- and 2-year survival rates showed no differences. Conclusion: CRS and HIPEC do not lead to an increased postoperative morbidity and mortality in AGC with PC. Albeit the poorer prognosis of patients with PC, survival of both groups was comparable.

C) 2021 The Author(s)

Published by S. Karger AG, Basel

\section{Introduction}

Gastric cancer is a worldwide healthcare burden, being the sixth most commonly diagnosed cancer and the third leading cause of cancer-related death in 2020 [1]. The combination of late onset of symptoms and the absence of a screening program in most countries often results in an advanced disease stage at the time of diagnosis. Therefore, $39 \%$ of patients are diagnosed at a locally advanced tumor stage, while $40 \%$ already show distant metastatic spread that goes hand-in-hand with a dramatically reduced life expectancy [2]. The peritoneum is a frequent metastatic site, especially for the diffuse subtype of gastric cancer, occurring in approximately $55 \%$ of patients [3]. The median overall survival (OS) of patients with untreated peritoneal carcinomatosis (PC) amounts to 3 months [4]. Although palliative chemotherapy has been shown to prolong survival in patients with distant metastases (i.e., in the liver and/or lung) up to 11-13 months, the benefit for patients suffering from PC is unclear $[5,6]$. 
In addition, the presence of $\mathrm{PC}$ has a higher influence on the quality of life of patients compared to other metastatic sites due to often occurring abdominal pain, severe ascites, and ileus [7].

Within the last decade, the combined therapeutic approach of cytoreductive surgery (CRS) with hyperthermic intraperitoneal chemotherapy (HIPEC) has been established. Since the technique was first described in the 1990 s, it is nowadays frequently performed in specialized surgical centers [8]. CRS and HIPEC has increased the OS of gastric cancer patients with PC up to 10-16 months [9-11]. Some studies suggest that this benefit is at the expense of increased postoperative complications, adding to the already high rate of severe complications after gastrectomy alone of up to $36.7 \%$ of all cases [12]. An increase in the frequency of the grave complication anastomotic leakage as well as wound infections after HIPEC has been described [13-15]. Studies investigating postoperative complications after HIPEC are scarce, but urgently needed for evidence-based decision-making. As important as documenting is the scoring of complications in a systematic manner. To this end, the Clavien-Dindo Classification (CDC), a well-established scoring system for postoperative complications, can be used [16]. To summarize all incurred complications, the Comprehensive Complication Index (CCI) has been established. The $\mathrm{CCI}$ is a single score that reflects the total sum of weighted single complications' severity [17]. The purpose of this study was to investigate the complications associated with CRS and HIPEC and to compare overall and disease-free survival to gastrectomy alone.

\section{Materials and Methods}

\section{Statement of Ethics}

The study was approved by the Ethics Committee of the Technical University of Dresden (BO-EK-431092020). All procedures performed in this study involving human participants were in accordance with the 1964 Declaration of Helsinki and its later amendments.

\section{Patient Selection}

All patients, who underwent gastrectomy with CRS and HIPEC in the University Hospital of the Technical University Dresden between 2013 and 2019 that did not meet any exclusion criteria, were included in the study (HIPEC group, $n=15$ ). Exclusion criteria were defined as a previous/concurrent second malignancy, previous extensive abdominal surgery, and substantial medical comorbidities like acute pancreatitis or coronary heart disease with instable angina pectoris. The control group included all consecutive patients that received a curative intent gastrectomy from the same time period due to locally advanced gastric cancer (pT3-4, pN1-3) but without PC and not meeting exclusion criteria (non-PC group, $n=43$ ).

Performed Treatments Including Surgery and HIPEC

Neoadjuvant treatment was performed in case clinical staging indicated a primary tumor of $\mathrm{T}>2$ and/or presence of lymph node metastases. Primary workup included a gastroscopy with histological confirmation and a CT scan of the thorax and abdomen. In case neoadjuvant chemotherapy was planned, a laparoscopy was also part of the primary workup. Primary tumor resection was performed according to histological subtype and location: diffuse type cancer was resected with a gastrectomy and intestinal type cancer was resected with minimum $8-\mathrm{cm}$ distance to the tumor, resulting in subtotal resections in case parts of the proximal corpus and/or fundus could be preserved. The extent of surgery in both groups included a subtotal, total, or transhiatal extended gastrectomy via laparotomy with a lymphadenectomy depending on tumor location. If necessary, a multivisceral resection with splenectomy, (left) pancreatectomy, and/or hemicolectomy was conducted. All patients were reconstructed with a Roux-Y gastro- or esophago-jejunostomy.

The decision to perform CRS and HIPEC was based on a PCI $\leq 12$ and the possibility to reach a (near) total cytoreduction (Completeness of Cytoreduction Score of $0-1$ ). CRS and HIPEC was not indicated if other nonresectable distant metastases existed or if patients were unfit for surgical resection or had known contraindication to intraperitoneal chemotherapy. The Peritoneal Cancer Index (PCI) was calculated after laparotomy in patients with PC according to Sugarbaker [18]. In the HIPEC group, CRS was performed by additional peritonectomy of PC lesions with the goal of achieving a complete cytoreduction. In 10 patients, the oncological resection was directly followed by HIPEC. In the remaining 5 patients, HIPEC was applied depending on the extent of surgery in a 2-staged procedure 5-6 days following the primary resection. Routinely, 2 afferent and 3 efferent drainages were placed and connected to the HIPEC pumping system. In 14 patients, HIPEC was performed with i.p. oxaliplatin $\left(350 \mathrm{mg} / \mathrm{m}^{2}\right)$ heated to $41.5^{\circ} \mathrm{C}$ $\left( \pm 0.5^{\circ} \mathrm{C}\right)$ for $30 \mathrm{~min}$ and an i.v. injection of $5-\mathrm{FU}\left(400 \mathrm{mg} / \mathrm{m}^{2}\right)$ and calciumfolinat $\left(20 \mathrm{mg} / \mathrm{m}^{2}\right)$. In 1 case, the patient refused systemic chemotherapeutic treatment, and therefore doxorubicin $(15 \mathrm{mg} /$ $\left.\mathrm{m}^{2}\right)$ and cisplatin $\left(50 \mathrm{mg} / \mathrm{m}^{2}\right)$ were applied i.p. for $60 \mathrm{~min}$ with a temperature of $41.0^{\circ} \mathrm{C}$.

\section{Morbidity Grading}

The analysis of postoperative complications was performed by 2 independent physicians by a systematic inspection of patient charts and medical files. In total, 17 frequent and severe postoperative complications after gastrectomy were evaluated: anastomotic leakage, duodenal stump insufficiency, pancreatic fistula, bleeding, intra-abdominal abscess, wound infection, wound dehiscence, (paralytic) ileus, pneumonia, respiratory insufficiency, prolonged ICU stay, arrhythmia, renal insufficiency, thromboembolic events, cardiac decompensation, bacteremia/sepsis, and multiple organ failure $[19,20]$. The extended CDC of the "Japan Clinical Oncology Group" was used to quantify postoperative morbidity [21]. All of the aforementioned complications that occurred within the first 90 postoperative days and were related to surgery were recorded. Next, the complications were weighted according to the CCI.

\section{Follow-Up}

Patients were followed up according to a standardized protocol at our University Cancer Center (UCC Dresden). This included a periodic clinical and imaging follow-up after surgery or completed adjuvant chemotherapy with CT scan and endoscopy every 6 months for 5 years. If necessary, a further diagnostic evaluation (e.g., endoscopic biopsy and re-laparotomy) was performed.

\section{Statistical Analysis}

To determine statistical differences between both groups, Fisher's exact test was used. The evaluation was performed with Student's $t$ test for metric, normal distributed variables and with the
Merboth/Garcia/v. Renesse/Distler/ Welsch/Weitz/Stange 
Table 1. Patient characteristics and operative and pathological findings

\begin{tabular}{|c|c|c|c|}
\hline & HIPEC, $n=15$ & Non-PC, $n=43$ & $p$ value \\
\hline Age, years & $59.4(50.1-69.3)$ & $63.6(57.6-74.8)$ & $0.091^{*}$ \\
\hline \multicolumn{4}{|l|}{ Sex } \\
\hline Female & $8(53.3)$ & $12(27.9)$ & \multirow[t]{2}{*}{$0.073^{\dagger}$} \\
\hline Male & $7(46.7)$ & $31(72.1)$ & \\
\hline BMI & $23.3(21.9-26.0)$ & $24.2(22.8-27.4)$ & $0.276^{*}$ \\
\hline Smoking & $3(20.0)$ & $10(23.3)$ & $0.552^{\dagger}$ \\
\hline Alcohol & $1(6.7)$ & $3(7.0)$ & $0.727^{\dagger}$ \\
\hline Diabetes mellitus & $0(0.0)$ & $8(18.6)$ & $0.076^{\dagger}$ \\
\hline \multicolumn{4}{|l|}{ Localization } \\
\hline AEG III/cardia & $1(6.7)$ & $12(27.9)$ & \multirow[t]{3}{*}{$1.000^{\dagger}$} \\
\hline Corpus & $8(53.3)$ & $21(48.8)$ & \\
\hline Antrum/pylorus & $6(40.0)$ & $10(23.3)$ & \\
\hline \multicolumn{4}{|l|}{ Lauren classification } \\
\hline Intestinal & $4(26.7)$ & $19(44.2)$ & \multirow[t]{3}{*}{$0.384^{\dagger}$} \\
\hline Mixed & $2(13.3)$ & $7(16.3)$ & \\
\hline Diffuse & $9(60.0)$ & $15(34.9)$ & \\
\hline \multicolumn{4}{|l|}{ Differentiation grade } \\
\hline Differentiated & $6(40.0)$ & $17(39.5)$ & \multirow[t]{2}{*}{$0.604^{\dagger}$} \\
\hline Undifferentiated & $9(60.0)$ & $29(60.5)$ & \\
\hline Signet ring cell & $9(60.0)$ & $11(25.6)$ & $0.019^{\dagger}$ \\
\hline \multicolumn{4}{|l|}{ pT stage } \\
\hline 3 & $9(60.0)$ & $30(69.8)$ & \multirow[t]{3}{*}{$0.423^{+}$} \\
\hline $4 a$ & $5(33.3)$ & $7(16.3)$ & \\
\hline $4 b$ & $1(6.7)$ & $6(14.0)$ & \\
\hline \multicolumn{4}{|l|}{ pN stage } \\
\hline 1 & $4(26.7)$ & $12(27.9)$ & \multirow[t]{4}{*}{$0.666^{\dagger}$} \\
\hline 2 & $4(26.7)$ & $13(30.2)$ & \\
\hline $3 a$ & $5(33.3)$ & $8(18.6)$ & \\
\hline $3 b$ & $2(13.3)$ & $10(23.3)$ & \\
\hline \multicolumn{4}{|l|}{ pM stage } \\
\hline 0 & $0(0.0)$ & $39(90.7)$ & \multirow[t]{2}{*}{$<0.001^{\dagger}$} \\
\hline 1 & $15(100.0)$ & $4(9.3)$ & \\
\hline \multicolumn{4}{|l|}{ M1 status } \\
\hline Peritoneum & $15(100.0)$ & $0(0.0)$ & \multirow[t]{2}{*}{$<0.001^{\dagger}$} \\
\hline Liver & $1(6.7)$ & $4(9.3)$ & \\
\hline \multicolumn{4}{|l|}{ UICC stage } \\
\hline IIB & $0(0.0)$ & 9 (20.9) & \multirow[t]{5}{*}{$<0.001^{\dagger}$} \\
\hline IIIA & $0(0.0)$ & $3(7.0)$ & \\
\hline IIIB & $0(0.0)$ & $9(20.9)$ & \\
\hline IIIC & $0(0.0)$ & $7(16.3)$ & \\
\hline IV & $15(100.0)$ & $4(9.3)$ & \\
\hline CTx neoadjuvant & $13(87.7)$ & $27(62.8)$ & $0.077^{\dagger}$ \\
\hline CTx adjuvant & $9(64.3)$ & $28(70.0)$ & $0.467^{\dagger}$ \\
\hline \multicolumn{4}{|l|}{ Gastrectomy } \\
\hline Subtotal & $2(13.3)$ & $12(27.9)$ & $0.449^{\dagger}$ \\
\hline Total & $10(66.7)$ & $21(48.8)$ & \\
\hline Transhiatal extended & $3(20.0)$ & $10(23.3)$ & \\
\hline Extended resection & $6(40.0)$ & $13(30.2)$ & $0.384^{\dagger}$ \\
\hline Splenectomy & $2(13.3)$ & $1(2.3)$ & \\
\hline Multivisceral & $2(13.3)$ & $7(16.3)$ & \\
\hline Others & $2(13.3)$ & $5(11.6)$ & \\
\hline Lymph nodes & & & \\
\hline Resected & $27(18-36)$ & $27(22-37)$ & $0.276^{\#}$ \\
\hline Infiltrated & $6(2-14)$ & $6(2-14)$ & $0.463^{\#}$ \\
\hline R1 status & $5(33.3)$ & $4(9.3)$ & $\mathbf{0 . 0 4 1 ^ { \dagger }}$ \\
\hline $\mathrm{PCl}$ & $7(3-10)$ & 0 & $0.000^{\#}$ \\
\hline
\end{tabular}

Values are $n$ (\%). All continuous numbers are median values with the interquartile range (IQR) in brackets. Bold values are significant. BMI, body mass index; $A E G$, adenocarcinoma of the esophagogastric junction; $\mathrm{PCl}$, Peritoneal Carcinomatosis Index; CTx, chemotherapy; UICC, Union internationale contre le cancer. ${ }^{\dagger}$ Fisher's exact test. ${ }^{*} t$ test. ${ }^{*}$ Mann-Whitney $U$. 
Table 2. Morbidity and mortality

\begin{tabular}{llll}
\hline & HIPEC, $n=15$ & Non-PC, $n=43$ & $p$ value \\
\hline CDC $\geq$ Illa & $4(26.7)$ & $11(25.6)$ & $0.592^{\dagger}$ \\
CCI (mean \pm SD) & $19.4(28.4)$ & $18.1(29.0)$ & $0.268^{\#}$ \\
Surgical complications & & & \\
$\quad$ Anastomotic leakage & $1(6.7)$ & $4(9.3)$ & $0.790^{\dagger}$ \\
$\quad$ Duodenal stump insufficiency & $0(0.0)$ & $1(2.3)$ & $0.741^{\dagger}$ \\
$\quad$ Intra-abdominal abscess & $2(13.3)$ & $4(9.3)$ & $0.703^{\dagger}$ \\
$\quad$ Wound infection & $3(20.0)$ & $4(9.3)$ & $0.408^{\dagger}$ \\
$\quad$ Bleeding & $1(6.7)$ & $3(7.0)$ & $0.727^{\dagger}$ \\
Medical complications & $1(6.7)$ & $3(7.0)$ & \\
$\quad$ Bacteremia/sepsis & $1(6.7)$ & $1(2.3)$ & $1.000^{\dagger}$ \\
$\quad$ Pneumonia & $1(6.7)$ & $6(14.0)$ & $0.454^{\dagger}$ \\
$\quad$ Thromboembolic events & $2(13.3)$ & $7(16.3)$ & $0.840^{\dagger}$ \\
$\quad$ Respiratory insufficiency & $15(13-20)$ & $13(9-19)$ & $0.462^{\dagger}$ \\
Length of stay, days & $2(13.3)$ & $6(14.0)$ & $0.099^{\#}$ \\
Reoperation & $1(7.1)$ & $4(9.3)$ & $0.662^{\dagger}$ \\
Readmission & $1(6.7)$ & $2(4.7)$ & $0.614^{\dagger}$ \\
30-day mortality & & & $0.600^{\dagger}$ \\
\hline
\end{tabular}

Values are $n$ (\%) unless otherwise indicated. CDC, Clavien-Dindo Classification; $\mathrm{CCl}, \mathrm{Com}$ prehensive Complication Index; SD, standard deviation. ${ }^{\dagger}$ Fisher's exact test. ${ }^{*}$ Mann-Whitney U.

Mann-Whitney U test for nonparametric variables. OS and disease-free survival (DFS) were analyzed with the Cox-regression and log-rank test and were represented as Kaplan-Meier curves. Significance level was set to 0.05 . The entire statistical analysis was performed in SPSS 25 (SPSS Statistics, v25.0.0.2; IBM Corporation, Armonk, NY, USA).

The manuscript is STROBE compliant. For STROBE checklist, please see online supplementary material (for all online suppl. material, see www.karger.com/doi/10.1159/000520330).

\section{Results}

Patient Characteristics, Pathological Findings, and Operative Procedures

In total, 58 patients with locally advanced gastric cancer that underwent subtotal, total, or transhiatal extended gastrectomy with lymphadenectomy were analyzed. Fifteen patients with PC received additional CRS and HIPEC. The control group consisted of a cohort of 43 patients with advanced gastric cancer without PC. Baseline characteristics such as age, sex, risk factors, and tumor localization were not statistically different in the 2 groups (Table 1). No difference in histological findings, that is, Lauren classification or differentiation grade, was found. As expected, the amount of signet ring cells as defined by the WHO (nucleus pressed to the cell edge, PASpositive intracellular mucus) was significantly higher in the HIPEC group $(60.0 \%$ vs. $25.6 \%, p=0.019)$. The pathological findings for $\mathrm{T}$ and $\mathrm{N}$ stage did not differ. Four patients in the non-PC group showed concomitant liver metastasis, which were resected in sano by atypical liver resection. A trend toward more neoadjuvant chemother- apy in the HIPEC group was detected ( $87.7 \%$ vs. $62.8 \%$, $p=0.077$ ), while the frequency of administered adjuvant chemotherapy was similar $(64.3 \%$ vs. $70.0 \%, p=0.467)$. Reasons for not administering neoadjuvant chemotherapy were old age with comorbidities or reduced condition $(n=9)$, primary resection requested by the patient $(n=$ $5)$, no indication based on preoperative staging $(n=3)$, and pyloric stenosis with inability of food intake $(n=1)$. Reasons for not administrating adjuvant treatment were old age with comorbidities or reduced condition $(n=7)$, refusal of chemotherapy $(n=7), 8$ or more cycles of preoperative chemotherapy, and therefore no indication for additional postoperative treatment $(n=2)$, and change to palliative situation due to tumor progress $(n=1)$ (online suppl. Table 1). The surgical procedures including lymph node yield were distributed similarly between the 2 treatment groups with the exception of a higher proportion of patients with a positive resection margin (R1) in the HIPEC group $(33.3 \%$ vs. $9.3 \%, p=0.041)$. In all patients of the HIPEC group, a Sugarbaker completeness of cytoreduction score (CCS) of $0-1$ could be achieved. Median PCI was 7 (IQR 3-10) in the HIPEC group (Table 1).

\section{Morbidity and Mortality}

Complications were considered serious if they required a surgical, endoscopic, or radiological intervention or intensive care (CDC score $\geq \mathrm{IIIa}$ ). Approximately one-quarter of the patients in both groups had one or more complications with a CDC score $\geq \mathrm{IIIa}(26.7 \%$ vs. $25.6 \%, p=0.592$ ) (Table 2; online suppl. Table 2). Likewise, the mean CCI (weighted sum of all complications) did not differ between both groups (19.4 vs. 18.1, $p=$
Merboth/Garcia/v. Renesse/Distler/ Welsch/Weitz/Stange 
Table 3. Recurrence and survival

\begin{tabular}{llll}
\hline & HIPEC, $n=14$ & Non-PC, $n=40$ & $p$ value \\
\hline Recurrence & $12(85.7)$ & $26(65.0)$ & $0.130^{\dagger}$ \\
$\quad$ Lymph nodes & $3(21.4)$ & $12(30.0)$ & $0.404^{\dagger}$ \\
$\quad$ Hematogenous & $3(21.4)$ & $13(32.5)$ & $0.337^{\dagger}$ \\
$\quad$ Peritoneal & $8(57.1)$ & $15(37.5)$ & $0.167^{\dagger}$ \\
$\quad$ Locoregional & $1(7.1)$ & $8(20.0)$ & $0.254^{\dagger}$ \\
$\quad$ Others & $3(21.4)$ & $4(10.0)$ & $0.253^{\dagger}$ \\
Time to first recurrence, months & $8.5(4-17.5)$ & $7.5(4-13)$ & $0.497^{\#}$ \\
Time to PC recurrence, months & $8.5(4-23)$ & $8(5-13)$ & $0.621^{\#}$ \\
Survival & & & \\
$\quad$ 1 year & $9(60.0)$ & $29(67.4)$ & $0.850^{\dagger}$ \\
$\quad$ 2 years & $4(26.7)$ & $13(30.2)$ & \\
Median survival (surgery), months & $12(9-26)$ & $15(9-32)$ & $0.538^{\#}$ \\
Median survival (diagnosis), months & $21(14-30)$ & $18(13-34)$ & $0.879^{\#}$ \\
\hline
\end{tabular}

Values are $n(\%)$. All continuous numbers are median values with the interquartile range (IQR) in brackets. PC, peritoneal carcinomatosis. ${ }^{\dagger}$ Fisher's exact test. ${ }^{*}$ Log-rank test.

0.268). We also did not detect significant difference between the groups, when analyzing the most frequent and severe surgical or medical postoperative complications individually. Although there were twice as many wound infections in the HIPEC group, this difference turned out to be not significant $(20.0 \%$ vs. $9.3 \%, p=0.408)$. The number of reoperations $(13.1 \%$ vs. $14.0 \%, p=0.662)$ and 90 day postoperative readmission rate $(7.1 \%$ vs. $9.3 \%, p=$ $0.614)$ were similar in both groups. The addition of CRS and HIPEC did not increase the 30 -day mortality $(6.7 \%$ vs. $4.7 \%, p=0.600$ ). Of note, in 2 patients, adverse events directly associated with HIPEC were observed: one allergic reaction that led to immediate termination of the HIPEC procedure and one pancytopenia, which resulted in septic multiple organ failure and death.

\section{Recurrence and Survival Analysis}

The total recurrence rate $(85.7 \%$ vs. $65.0 \%, p=0.130)$ and the rate of peritoneal recurrences $(57.1 \%$ vs. $37.5 \%$, $p=0.167)$ were each approximately $20 \%$ higher in the HIPEC group, but proved to not be statistically significantly different (Table 3 ). The median time between surgery and first recurrence was 8.5 months (4-17.5 months) in the HIPEC group and 7.5 months (4-13 months) in the non-PC group (HR 0.808, 95\% CI: 0.382-1.709, $p=$ $0.578)$. Of note, the time between surgical resection and first peritoneal recurrence was similar in both groups ( 8.5 months [4-23 months] vs. 8.0 [5-13 months], HR 0.643, 95\% CI: $0.241-1.715, p=0.377)$. The 1 - and 2-year survival was $60.0 \%$ and $26.7 \%$ in the HIPEC group and $67.4 \%$ and $30.2 \%$ in the non-PC group ( $p=0.850$ ). The median OS time after surgery was 12 months (9-26 months) in the HIPEC group and 15 months (9-32 months) in the non-PC group (HR 1.240, 95\% CI: $0.616-2.495, p=$ $0.546)$. The median OS time from date of diagnosis was 21 months (14-30 months) in the HIPEC group and 18 months (13-24 months) in the non-PC group (HR 1.054, 95\% CI: 0.524-2.121, $p=0.882$ ). No difference in KaplanMeier curves for OS $(p=0.538)$ and DFS for PC ( $p=$ 0.558 ) after 2 years of follow-up was observed (Fig. 1).

Within the HIPEC group, 4 patients with PCI $\leq 6(n=$ 7) and 4 patients with PCI $>6(n=7)$ developed a PC recurrence $(57.1 \%$ vs. $57.1 \%, p=0.704)$. While the recurrence rates in both groups were similar, the median survival of patients with lower PCI was significantly different with 20 months (12-47 months) compared to 9 months (7-14 months, $p=0.015)$ in patients with higher PCI. In line with this, Kaplan-Meier curves documented a clear trend toward a difference in OS depending on PCI ( $p=$ 0.051) (Fig. 2).

\section{Discussion}

The "peritoneal-blood-barrier" between the submesothelial tissue and peritoneal surface limits nutrient and oxygen transport. This barrier function is thought to reduce the effectiveness of systemic chemotherapy [22,23]. Based on this hypothesis, the concept of intraperitoneal chemotherapy was developed and nowadays performed in conjunction with hyperthermia (HIPEC). Nevertheless, the HIPEC procedure is still controversially discussed. One of the frequently voiced fears is the increased morbidity and mortality of CRS and HIPEC in addition to the already major surgery of the primary tumor. Mortality is reported to range between $5.2 \%$ and $11.7 \%$ after gastrectomy $[12,19]$ and $3 \%$ and $10 \%$ after CRS and HIPEC for gastric cancer [24]. In accordance with this, we found a mortality rate of $6.7 \%$ and $4.7 \%$ in the HIPEC and non-PC groups, respectively. The morbidity of total or subtotal gastrectomy for cancer varies from $17 \%$ to $43 \%$ [25], and major postoperative complications (CDC 
Fig. 1. OS and DFS for PC in both groups (Kaplan-Meier curves, log-rank test). OS, overall survival; DFS, disease-free survival; PC peritoneal carcinomatosis; HIPEC, hyperthermic intraperitoneal chemotherapy.

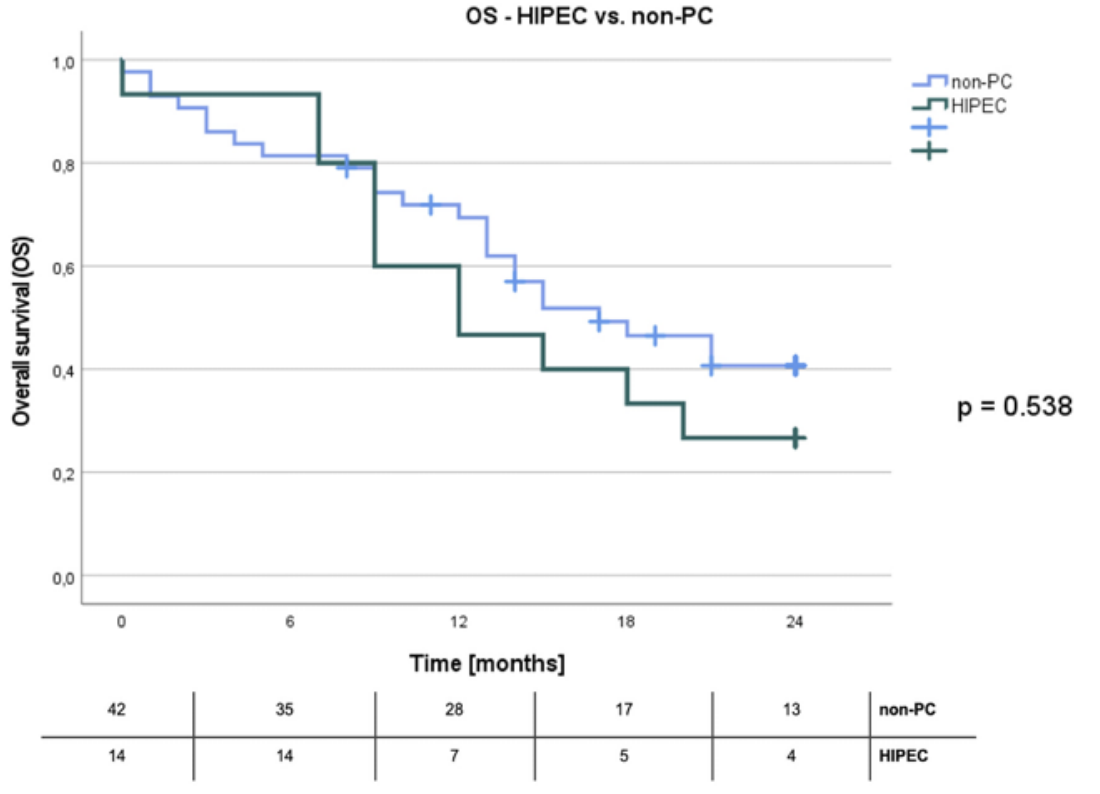

DFS for PC - HIPEC vs. non-PC

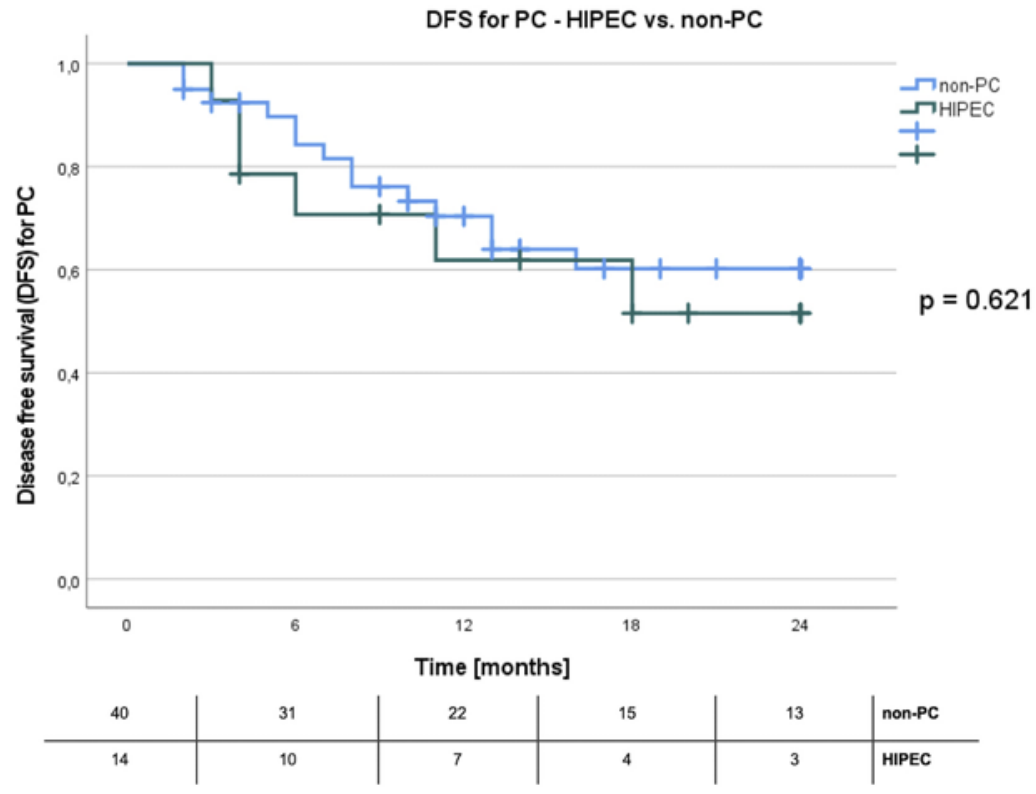

$\geq$ IIIa) are reported to occur in $9.8-18.9 \%$ of all cases [ 15 , 26]. The mean CCI after gastrectomy ranges from 5.8 to 9.4 in the literature [27, 28]. Mielko et al. [29] reported higher morbidity rates after CRS and HIPEC with a mean CCI of 42.7 (SD 22.7). We observed one or more postoperative complications with $\mathrm{CDC} \geq$ IIIa in approximately one-quarter of patients in both the HIPEC and the nonPC group, and the mean CCI was 19.4 and 18.1, respectively. Of note, we have included 17 distinct complications into our analysis, surpassing the usual 8-10 complications taken into account by previous publications. While the CCI for the HIPEC group was thus better than what has been published before, the frequency of compli- cations with CDC $\geq$ IIIa was above the frequency previously reported for total or subtotal gastrectomy. However, our non-PC cohort included only advanced gastric cancer (pT3-4, pN1-3), and one-third of patients underwent extended resections, which influences the frequency and severity of morbidity.

When focusing on individual postoperative morbidities, a large analysis of 37,440 German patients, which received subtotal or total gastrectomy, revealed morbidity rates for anastomotic leakage of $9.7 \%$, pneumonia of $12.3 \%$, and sepsis of $18.3 \%$ [12]. In our non-PC group, the rates of these complications are in part distinctly lower with $9.3 \%, 2.3 \%$, and $7.0 \%$, respectively. In the literature, 
Fig. 2. OS depending on PCI in the HIPEC group (Kaplan-Meier curves, log-rank test). OS, overall survival; HIPEC, hyperthermic intraperitoneal chemotherapy; PCI, Peritoneal Cancer Index.

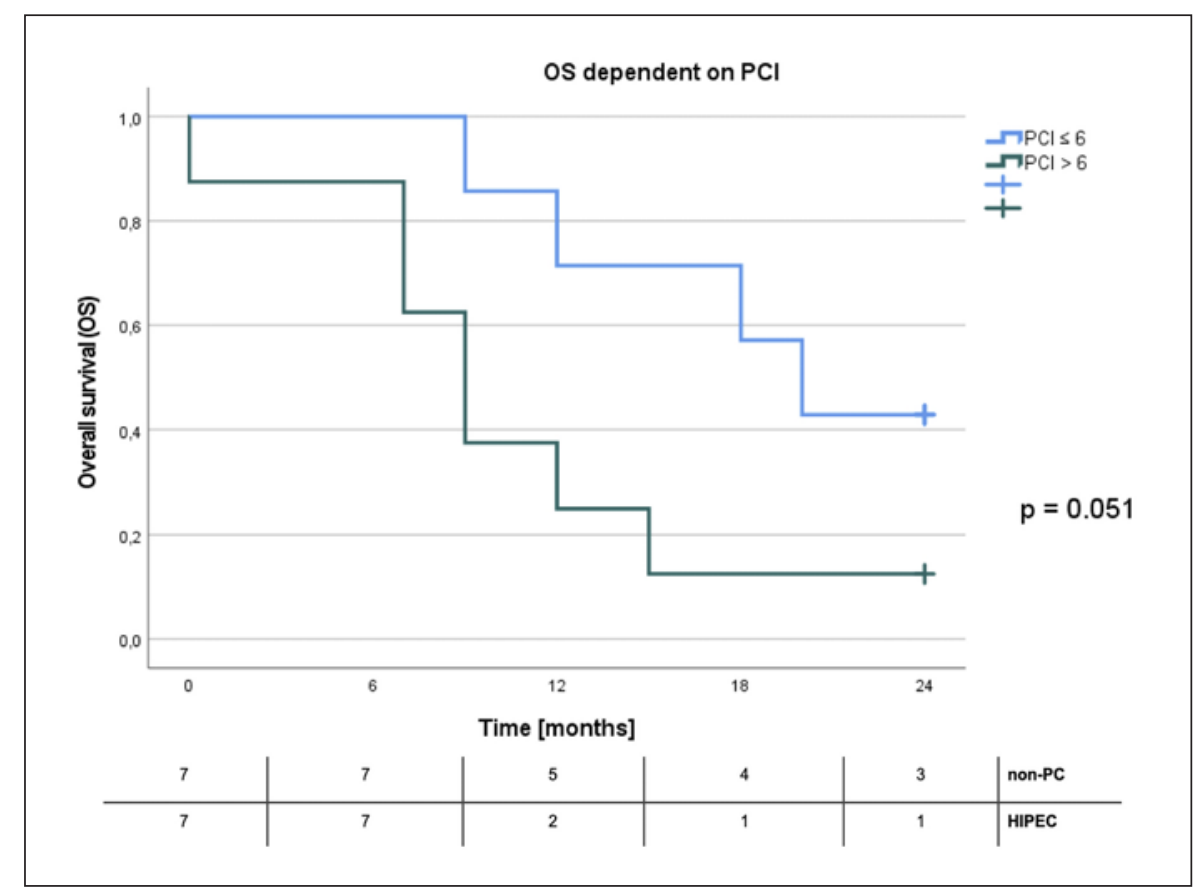

an increased risk after HIPEC for developing an intraabdominal abscess, respiratory failure, or renal dysfunction is described $[24,30]$. In our analyses, no significant differences in surgical and medical complications between the HIPEC and non-PC group were found. Thus, no increase in the frequency of CDC $\geq \mathrm{III}$ a and CCI nor in individual morbidities was seen in our comparison. This is of importance, as it has been argued that performing CRS and HIPEC delays additive chemotherapy because of postoperative complications and a general longer recovery time after surgery [24]. We could demonstrate for our patient cohorts no significant difference in the rate of administered adjuvant chemotherapy.

In patients with advanced gastric cancer and curative resection, the most common site of recurrence is the peritoneum with approximately 39\% [31]. Consistent with this, we found a PC recurrence rate in our control cohort of $37.5 \%$. The median time for developing a PC recurrence was 8.5 months in the HIPEC group and similarly 8 months in the non-PC group. The recurrence pattern after CRS and HIPEC is not well examined. Rosa et al. [32] showed a PC recurrence rate of $57 \%$ after CRS and HIPEC for primary PC and of 59\% after surgery alone in advanced gastric cancer. In our HIPEC cohort, $57.1 \%$ patients developed a peritoneal recurrence. In other words, this implicates that in $42.9 \%$ of the patients, PC did not return until death or within the follow-up period. CRS and HIPEC thus results in a similar time interval to PC recurrence between patients with and without $\mathrm{PC}$, while a large proportion does not experience a relapse. This is of importance, as the quality of life of patients declines in the presence of untreated PC due to often occurring ab- dominal pain, severe ascites, and ileus [33]. A systemic review could demonstrate that the quality of life measured by standardized questionnaires initially decreases after CRS and HIPEC, but recovers to preoperative levels by $6-12$ months after surgery [34]. Moreover, it could be shown that patients suffering from symptomatic malignant ascites profit the most from CRS and HIPEC in terms of quality of life [35].

Several studies have indicated positive effects of CRS and HIPEC in gastric cancer patients [36, 37]. In a large multi-institutional study, Glehen et al. [38] could document in 159 patients with gastric cancer and PC treated with HIPEC and/or early postoperative intraperitoneal chemotherapy a median OS of 9.2 months and a 1-year survival of $43 \%$. The cohort included patients with high PCI of $>19$. In a subgroup analysis of patients with CCS $0-1$, the median survival was 15 months. Up to today, Yang and colleagues [9] performed the only larger randomized controlled trial comparing CRS and HIPEC to CRS alone for gastric cancer with PC. A significantly better median OS of 11.0 months in comparison with 6.5 months for the CRS and HIPEC group versus CRS alone was described. The 1- and 2-year survival rates were $41.2 \%$ and $14.7 \%$ for CRS and HIPEC and $24.9 \%$ and $5.9 \%$ for CRS alone [9]. In a meta-analysis, 289 patients undergoing CRS and HIPEC for gastric cancer and PC were evaluated revealing a median OS of 11.1 months [24]. With a median OS of 12 months and 1- and 2-year survival rates of $60.0 \%$ and $26.7 \%$, we observed similar results in the present study within the HIPEC group. Of note, we could document in our analysis that the life expectancy of patients with primary PC after CRS and 
HIPEC did not differ from resected patients without PC (median OS both from surgery and from initial diagnosis) (Table 3).

An optimal PCI cutoff for CRS and HIPEC is controversially discussed in the literature [8]. A large meta-analysis of 748 patients defined a PCI of 12 as an optimal PCI cutoff [39]. Other authors favor a lower PCI cutoff of $\leq 6$ $[29,40]$. We also observed a better OS in the group of patients with $\mathrm{PCI} \leq 6$, but $\mathrm{PC}$ recurrence rates showed to be independent of the PCI value: in both patient groups, $42.9 \%$ did not suffer from a PC recurrence within the follow-up period. Our results favor the idea that also patients with PCI $>6$ could possibly benefit from CRS and HIPEC due to the negative impact of PC on the quality of life.

There are clear limitations of this study, that is, the retrospective, not randomized analysis, the small number of patients that also prohibited a matching of the control group, and the short follow-up of only 2 years. Furthermore, all patients were treated at a single institution, limiting the generalizability of the results. On the other hand, we rigorously analyzed the postoperative course of the patients included in the study, implementing up-to-date measures for postoperative complications. Based on our data, we thus believe to be able to conclude that CRS and HIPEC with primary PC is a safe procedure that does not increase morbidity and mortality rates compared to nonPC patients with similar advanced gastric cancer ( $\mathrm{pT} 3-4$, pN1-3, UICC III or higher), at least in our hospital setting. The finding of a high percentage of patients with PCI $>6$ not suffering from a PC recurrence is noteworthy. We hypothesize that quality of life is improved by CRS and HIPEC also in the PCI $>6$ group of patients, as the absence of $\mathrm{PC}$ even in the setting of a high overall recurrence rate of $85.6 \%$ could prevent typical complications of PC. Furthermore, the OS of patients with primary PC after CRS and HIPEC was not different from patients with advanced gastric cancer without PC. In conclusion, we found no increase in morbidity and mortality after CRS and HIPEC including patients with a PCI up to $12 \mathrm{com}$ pared to a similar advanced patient cohort without PC in our hospital setting while achieving a similar OS.

\section{Statement of Ethics}

The study was approved by the Ethics Committee of the Technical University of Dresden (BO-EK-431092020). All procedures performed in this study involving human participants were in accordance with the 1964 Declaration of Helsinki and its later amendments. All patients gave their written informed consent to the operative procedures.

\section{Conflict of Interest Statement}

The authors have no conflicts of interest to declare.

\section{Funding Sources}

This study was performed without funding by any sponsor.

\section{Author Contributions}

D.E. Stange and F. Merboth conceived of the presented idea. F. Merboth developed the theory and performed the computations. F. Merboth, S. Garcia, and J. v. Renesse performed data collection and verified the analytical methods. J. Weitz, T. Welsch, and M. Distler encouraged F. Merboth to investigate especially the PCIdepended life expectancy and supervised the findings of this work. All authors discussed the results and contributed to the final manuscript.

\section{Data Availability Statement}

All data generated or analyzed during this study are included in this article and/or its online supplementary files. Further enquiries can be directed to the corresponding author.

\section{References}

1 Sung H, Ferlay J, Siegel RL, Laversanne M, Soerjomataram I, Jemal A, et al. Global cancer statistics 2020: GLOBOCAN estimates of incidence and mortality worldwide for 36 cancers in 185 countries. CA Cancer J Clin. 2021 Feb 4;71(3):209-49.

2 Brandl A, Pachmayr E, Gül-Klein S, Alberto $\mathrm{M}$, Thuss-Patience P, Rau B. [Surgical treatment of peritoneal metastases of gastric cancer]. Chirurg. 2018 Sep;89(9):66977.

3 Coccolini F, Fugazzola P, Ansaloni L, Sartelli M, Cicuttin E, Leandro G, et al. Advanced gastric cancer: the value of systemic and intraperitoneal chemotherapy. Acta Biomed. 2018 Dec 17;89(8-S):104-9.
4 Sadeghi B, Arvieux C, Glehen O, Beaujard AC, Rivoire M, Baulieux J, et al. Peritoneal carcinomatosis from non-gynecologic malignancies: results of the EVOCAPE 1 multicentric prospective study. Cancer. 2000 Jan 15; 88(2):358-63.

5 Bang YJ, Van Cutsem E, Feyereislova A, Chung HC, Shen L, Sawaki A, et al. Trastuzumab in combination with chemotherapy versus chemotherapy alone for treatment of HER2-positive advanced gastric or gastro-oesophageal junction cancer (ToGA): a phase 3 , open-label, randomised controlled trial. Lancet. 2010 Aug 28;376(9742):687-97.

6 Wagner AD, Syn NL, Moehler M, Grothe W, Yong WP, Tai BC, et al. Chemotherapy for advanced gastric cancer. Cochrane Database Syst Rev. 2017 Aug 29;8:CD004064.

7 Sugarbaker PH. Peritoneal metastases from gastrointestinal cancer. Curr Oncol Rep. 2018 Jun 8;20(8):62.

8 Sugarbaker PH. Management of peritoneal metastases - basic concepts. J BUON. 2015 May;20 Suppl 1:S2-11.

9 Yang XJ, Huang CQ, Suo T, Mei LJ, Yang GL, Cheng FL, et al. Cytoreductive surgery and hyperthermic intraperitoneal chemotherapy improves survival of patients with peritoneal carcinomatosis from gastric cancer: final results of a phase III randomized clinical trial. Ann Surg Oncol. 2011 Jun; 18(6):1575-81. 
10 Canbay E, Mizumoto A, Ichinose M, Ishibashi H, Sako S, Hirano M, et al. Outcome data of patients with peritoneal carcinomatosis from gastric origin treated by a strategy of bidirectional chemotherapy prior to cytoreductive surgery and hyperthermic intraperitoneal chemotherapy in a single specialized center in Japan. Ann Surg Oncol. 2014 Apr; 21(4):1147-52.

11 Rau B, Brandl A, Thuss-Patience P, Bergner F, Raue W, Arnold A, et al. The efficacy of treatment options for patients with gastric cancer and peritoneal metastasis. Gastric Cancer. 2019 Nov;22(6):1226-37.

12 Baum P, Diers J, Lichthardt S, Kastner C Schlegel N, Germer CT, et al. Mortality and complications following visceral surgery: a nationwide analysis based on the diagnostic categories used in German Hospital invoicing data. Dtsch Arztebl Int. 2019 Nov 1;116(44): 739-46.

13 Coccolini F, Montori G, Ceresoli M, Cima S, Valli MC, Nita GE, et al. Advanced gastric cancer: what we know and what we still have to learn. World J Gastroenterol. 2016 Jan 21 22(3):1139-59.

14 Quenet F, Elias D, Roca L, Goere D, Ghouti L, Pocard $\mathrm{M}$, et al. A UNICANCER phase III trial of hyperthermic intra-peritoneal chemotherapy (HIPEC) for colorectal peritoneal carcinomatosis (PC): PRODIGE 7. J Clin Oncol. 2018;36(18_Suppl):LBA3503.

15 Li SS, Udelsman BV, Parikh A, Klempner SJ, Clark JW, Roeland EJ, et al. Impact of postoperative complication and completion of multimodality therapy on survival in patients undergoing gastrectomy for advanced gastric cancer. J Am Coll Surg. 2020 Jun;230(6):91224.

16 Dindo D, Demartines N, Clavien PA. Classification of surgical complications: a new proposal with evaluation in a cohort of 6336 patients and results of a survey. Ann Surg. 2004 Aug;240(2):205-13.

17 Slankamenac K, Graf R, Barkun J, Puhan MA, Clavien PA. The comprehensive complication index: a novel continuous scale to measure surgical morbidity. Ann Surg. 2013 Jul; 258(1):1-7.

18 Jacquet P, Sugarbaker PH. Clinical research methodologies in diagnosis and staging of patients with peritoneal carcinomatosis. Cancer Treat Res. 1996;82:359-74.

19 Martin AN, Das D, Turrentine FE, Bauer TW, Adams RB, Zaydfudim VM. Morbidity and mortality after gastrectomy: identification of modifiable risk factors. J Gastrointest Surg. 2016 Sep;20(9):1554-64.

20 Baiocchi GL, Giacopuzzi S, Marrelli D, Reim D, Piessen G, Matos da Costa P, et al. International consensus on a complications list after gastrectomy for cancer. Gastric Cancer. 2019 Jan;22(1):172-89.
21 Katayama H, Kurokawa Y, Nakamura K, Ito $\mathrm{H}$, Kanemitsu Y, Masuda N, et al. Extended Clavien-Dindo classification of surgical complications: Japan Clinical Oncology Group postoperative complications criteria. Surg Today. 2016 Jun;46(6):668-85.

22 Los G, Mutsaers PH, van der Vijgh WJ, Baldew GS, de Graaf PW, McVie JG. Direct diffusion of cis-diamminedichloroplatinum(II) in intraperitoneal rat tumors after intraperitoneal chemotherapy: a comparison with systemic chemotherapy. Cancer Res. 1989 Jun 15;49(12):3380-4.

23 González-Moreno S. Advances in peritoneal surface oncology. Berlin, NY: Springer; 2007.

24 Desiderio J, Chao J, Melstrom L, Warner S, Tozzi F, Fong Y, et al. The 30-year experienceA meta-analysis of randomised and highquality non-randomised studies of hyperthermic intraperitoneal chemotherapy in the treatment of gastric cancer. Eur J Cancer. 2017 Jul;79:1-14.

25 Nakagawa M, Kojima K, Inokuchi M, Kato K, Sugita H, Otsuki S, et al. Identification of frequency, severity and risk factors of complications after open gastrectomy: retrospective analysis of prospectively collected database using the Clavien-Dindo classification. J Med Dent Sci. 2016;63(2-3):53-9.

26 Wang WJ, Li R, Guo CA, Li HT, Yu JP, Wang $\mathrm{J}$, et al. Systematic assessment of complications after robotic-assisted total versus distal gastrectomy for advanced gastric cancer: a retrospective propensity score-matched study using Clavien-Dindo classification. Int J Surg. 2019 Nov; $71: 140-8$.

27 Kim TH, Suh YS, Huh YJ, Son YG, Park JH, Yang JY, et al. The comprehensive complication index (CCI) is a more sensitive complication index than the conventional ClavienDindo classification in radical gastric cancer surgery. Gastric Cancer. 2018 Jan;21(1):17181.

28 Shimizu S, Saito H, Kono Y, Murakami Y, Shishido Y, Miyatani K, et al. The prognostic significance of the comprehensive complication index in patients with gastric cancer. Surg Today. 2019 Nov;49(11):913-20.

29 Mielko J, Rawicz-Pruszyński K, Skórzewska M, Ciseł B, Pikuła A, Kwietniewska M, et al. Conversion surgery with HIPEC for peritoneal oligometastatic gastric cancer. Cancers. 2019 Nov 2;11(11): 1715

30 Yan TD, Black D, Sugarbaker PH, Zhu J, Yonemura Y, Petrou G, et al. A systematic review and meta-analysis of the randomized controlled trials on adjuvant intraperitoneal chemotherapy for resectable gastric cancer. Ann Surg Oncol. 2007 Oct;14(10):2702-13.
31 Kim JH, Lee HH, Seo HS, Jung YJ, Park CH. Stage-specific difference in timing and pattern of initial recurrence after curative surgery for gastric cancer. Surg Oncol. 2019 Sep; 30:81-6.

32 Rosa F, Galiandro F, Ricci R, Di Miceli D, Longo F, Quero G, et al. Survival advantage of cytoreductive surgery and hyperthermic intraperitoneal chemotherapy (HIPEC) for advanced gastric cancer: experience from a Western tertiary referral center. Langenbecks Arch Surg. 2021 Jun;406(4):1071-80.

33 Tsilimparis N, Bockelmann C, Raue W, Menenakos C, Perez S, Rau B, et al. Quality of life in patients after cytoreductive surgery and hyperthermic intraperitoneal chemotherapy: is it worth the risk? Ann Surg Oncol. 2013 Jan; 20(1):226-32

34 Leimkuhler M, Hentzen J, Hemmer PHJ, Been LB, van Ginkel RJ, Kruijff S, et al. Systematic review of factors affecting quality of life after cytoreductive surgery with hyperthermic intraperitoneal chemotherapy. Ann Surg Oncol. 2020 Oct;27(10):3973-83.

35 McQuellon RP, Loggie BW, Fleming RA, Russell GB, Lehman AB, Rambo TD. Quality of life after intraperitoneal hyperthermic chemotherapy (IPHC) for peritoneal carcinomatosis. Eur J Surg Oncol. 2001 Feb;27(1):65-73.

$36 \mathrm{Kim}$ JY, Bae HS. A controlled clinical study of serosa-invasive gastric carcinoma patients who underwent surgery plus intraperitoneal hyperthermo-chemo-perfusion (IHCP). Gastric Cancer. 2001;4(1):27-33.

37 Gill RS, Al-Adra DP, Nagendran J, Campbell S, Shi X, Haase E, et al. Treatment of gastric cancer with peritoneal carcinomatosis by cytoreductive surgery and HIPEC: a systematic review of survival, mortality, and morbidity. J Surg Oncol. 2011 Nov 1;104(6):692-8.

38 Glehen O, Gilly FN, Arvieux C, Cotte E, Boutitie F, Mansvelt B, et al. Peritoneal carcinomatosis from gastric cancer: a multi-institutional study of 159 patients treated by cytoreductive surgery combined with perioperative intraperitoneal chemotherapy. Ann Surg Oncol. 2010 Sep;17(9):2370-7.

39 Coccolini F, Catena F, Glehen O, Yonemura Y, Sugarbaker PH, Piso P, et al. Complete versus incomplete cytoreduction in peritoneal carcinosis from gastric cancer, with consideration to PCI cut-off. Systematic review and meta-analysis. Eur J Surg Oncol. 2015 Jul; 41(7):911-9.

40 Manzanedo I, Pereira F, Rihuete Caro C, Pérez-Viejo E, Serrano Á, Gutiérrez Calvo A, et al. Cytoreductive surgery and hyperthermic intraperitoneal chemotherapy (HIPEC) for gastric cancer with peritoneal carcinomatosis: Multicenter Study of Spanish Group of peritoneal oncologic surgery (GECOP). Ann Surg Oncol. 2019 Aug;26(8):2615-21. 